\title{
Ensayo clínico aleatorizado sobre la administración de una solución de mantenimiento intravenosa hipotónica comparada contra una isotónica en pacientes pediátricos críticos
}

\author{
Hypotonic versus isotonic maintenance fluids in critically ill \\ pediatric patients: a randomized control trial
}

Dr. Facundo A. Jorro Barón ${ }^{a}$, Dra. Claudia N. Meregalli ${ }^{a}$ Dra. Valeria A. Romboláa, Dra. Cecilia Bolasell ${ }^{a}$,Farm. Vilma E. Pigliapoco ${ }^{b}$, Farm. Silvia E. Bartolettib ${ }^{b}$ Dr. Gustavo E. Debaisia

\section{RESUMEN}

Introducción. Las soluciones hipotónicas se han vinculado a la producción de hiponatremia iatrogénica.

Objetivos. Evaluar las variaciones en el sodio sérico $(\mathrm{NaS})$ tras la administración de una solución de mantenimientointravenosa isotónica ( $\mathrm{NaCl}$ al $0,9 \%$ en dextrosa al $5 \%$ ) en comparación con una solución de mantenimiento hipotónica ( $\mathrm{NaCl}$ al $0,45 \%$ en dextrosa al 5\%).

Material y métodos. Ensayo clínico aleatorizado, doble ciego y controlado. Se enrolaron pacientes pediátricos con una estadía esperada en la unidad de cuidados intensivos mayor de 24 horas, NaS normal y líquidos intravenosos $>80 \%$ de los líquidos totales de mantenimiento. La concentración sérica de Na se midió antes de colocar la solución de mantenimiento y al reducir la administración de ésta a $<80 \%$ del total del aporte.

Resultados. Se incorporaron 63 pacientes, que fueron asignados en forma aleatoria a recibir una solución de mantenimiento hipotónica $(n=32)$ o isotónica $(n=31)$. Las características basales fueron similares en ambos grupos. No hubo diferencias con respecto a la cantidad de solución administrada (grupo hipotónico $865 \pm 853 \mathrm{ml}$; grupo isotónico $778 \pm 649 \mathrm{ml}$, $p=0,654$ ) o el tiempo de infusión (grupo hipotónico: $24 \pm 10,8$ horas; grupo isotónico: $27,6 \pm 12,8$ horas, $p=0,231$ ). Se encontró una diferencia en el NaS luego de la administración de las soluciones de mantenimiento (grupo hipotónico: 137,8 $\pm 4,3 \mathrm{mmol} / \mathrm{L}$; grupoisotónico: $140,0 \pm 4,1 \mathrm{mmol} / \mathrm{L}, p=0,04)$. Ninguna de las dos soluciones de mantenimiento aumentó el riesgo de hiponatremia $(\mathrm{Na}<135 \mathrm{mmol} / \mathrm{L})$ o de hipernatremia (Na $>145 \mathrm{mmol} / \mathrm{L})$.

Conclusiones. Ambas soluciones de mantenimiento, en 24 horas de infusión, no aumentaron el riesgo de producir hiponatremia iatrogénica. Palabrasclave: soluciones intravenosas, hipotónica, isotónica, ensayo clínico.

http:/ /dx.doi.org/10.5546/aap.2013.281

\section{INTRODUCCIÓN}

Las soluciones de mantenimiento (SM) son necesarias para garantizar el suministro de agua y electrolitos en los pacientes que no pueden tomar líquidos por vía oral. La composición óptima de esas soluciones en los pacientes pediátricos sigue siendo desconocida.

En 1956, Holliday y Segar ${ }^{1}$ sugirieron que los electrolitos por vía intravenosa utilizados en las SM podrían ser similares a los contenidos en la leche materna ( $\mathrm{ClNa} 10$ $30 \mathrm{mmol} / \mathrm{L}$ ). La utilización de SM hipotónicas en los niños sanos no debería asociarse a ningún riesgo, pero puede tener consecuencias perjudiciales en los niños enfermos que tienen una capacidad limitada para excretar agua libre.

Desde la década de 1980, numerosas publicaciones describieron la aparición de morbilidad neurológica grave y muerte secundaria a la producción de hiponatremia iatrogénica. ${ }^{2-4} \mathrm{~A}$ pesar de la calidad y cantidad de las infusiones dadas, la concentración de sodio sérico $(\mathrm{NaS})$ por lo general se mantiene dentro de los límites normales (135$145 \mathrm{mmol} / \mathrm{L}$ ). La homeostasis del agua y el sodio es sostenida por el riñón a través de la excreción de agua libre. Se describieron numerosos estímulos para la secreción de la hormona antidiurética; muchos de ellos son frecuentes en los pacientes críticamente enfermos, como las 
infecciones de las vías respiratoria y digestiva, el dolor, las náuseas, el uso de opiáceos y el período posquirúrgico..$^{5-7}$ Por lo tanto, las SM hipotónicas que contienen grandes cantidades de agua libre podrían inducir hiponatremia iatrogénica en estos pacientes. Debido a un aumento en el informe de casos de hiponatremia iatrogénica relacionados con lesiones cerebrales permanentes y muerte encefálica, numerosos autores recomiendan actualmente el uso de soluciones isotónicas como SM para los pacientes pediátricos..$^{8-11}$ A pesar de la evidencia creciente de que las soluciones hipotónicas aumentan el riesgo de hiponatremia, hay pocos ensayos clínicos prospectivos que lo confirmen en los pacientes pediátricos. ${ }^{8}$ En nuestro país aún se utilizan en algunos centros $\mathrm{SM}$ que contienen $\mathrm{NaCl}$ al $0,18 \%$ a $0,3 \%$ (30 a $45 \mathrm{mmol} / \mathrm{L}$ ) en dextrosa al 5\%, sin controles de $\mathrm{NaS}$, tanto al inicio como durante la infusión de las soluciones. ${ }^{12}$

El objetivo principal de este ensayo clínico fue evaluar la diferencia en la NaS con la administración de dos SM de distinta tonicidad, $\mathrm{SM}$ isotónica (SMI) (ClNa al 0,9\% en dextrosa al $5 \%)$ y SM hipotónica (SMH) (ClNa al $0,45 \%$ en dextrosa al 5\%) por menos de 48 horas después del ingreso en una unidad de cuidados intensivos pediátricos (UCIP).

\section{MATERIAL Y MÉTODOS Protocolo}

Se realizó un estudio prospectivo, controlado, aleatorizado y doble ciego en la UCIP del Hospital General de Niños "Pedro de Elizalde" (HGNPE), entre el 1 de febrero y el 31 de octubre de 2011. Fueron elegibles los niños en edades comprendidas entre 1 mes y 18 años, con una estadía esperada en la UCIP > 24 horas, con NaS normal (135-145 mmol/L) medido al ingresar en la UCIP o después de la reanimación con líquidos intravenosos y la exigencia de $>80 \%$ de la administración total de líquidos de mantenimiento por vía intravenosa. Se excluyeron los pacientes con insuficiencia renal previa (creatinina sérica $>1 \mathrm{mg} / \mathrm{dl}$ en los niños $\leq 3$ años y creatinina sérica $>1,5 \mathrm{mg} / \mathrm{dl}$ en los niños $>3$ años), insuficiencia hepática con ascitis e hipertensión portal, insuficiencia suprarrenal, síndrome nefrótico o nefrítico, enfermedad de Kawasaki, anemia drepanocítica, diabetes insípida, enfermedad metabólica congénita, glucosa en plasma $>200 \mathrm{mg} / \mathrm{dl}$; los pacientes con requerimientos de nutrición parenteral total o hiperhidratación (pacientes con tratamiento oncológico o riesgo de lisis tumoral) y los readmitidos en la UCIP. Los pacientes en los que no se obtuvo una segunda muestra de $\mathrm{NaS}$ quedaron excluidos del estudio.

Los niños, después de que los padres dieron su consentimiento informado, fueron asignados en forma aleatoria en dos grupos: el grupo hipotónico $(\mathrm{SMH})$, que recibió la $\mathrm{SM}$ con una concentración de sodio de $77 \mathrm{mmol} / \mathrm{L}$, y el grupo isotónico (SMI), que recibió la SM con una concentración de sodio de $154 \mathrm{mmol} / \mathrm{L}$. La adición de potasio hasta $20 \mathrm{mmol} / \mathrm{L}$ en las SM de ambos grupos la decidió el médico tratante. Las concentraciones de glucosa fueron las mismas en ambos grupos (dextrosa al 5\%). Las SM se prepararon y enmascararon por los investigadores farmacéuticos. Las SM se hicieron bajo flujo vertical laminar de seguridad biológica de clase $2 X$ y se almacenaron en bolsas cerradas herméticas individuales a una temperatura de $2-8^{\circ} \mathrm{C}$, hasta su dispensación con el respectivo código de aleatorización. La cantidad de líquido se calculó por las normas del HGNPE, $1500 \mathrm{ml} /$ $\mathrm{m}^{2}$ si el peso del paciente era $>10 \mathrm{~kg}$ y $80 \mathrm{~mL} / \mathrm{kg}$ si el peso del paciente era $\leq 10 \mathrm{~kg}$, cada 24 horas.

El médico tratante pudo interrumpir el tratamiento con SM y retirar al paciente del protocolo en cualquier momento que considerara necesario realizar un cambio en la concentración de Na infundida. Los médicos tratantes conocían los resultados de la natremia de los pacientes ingresados en el estudio para poder controlar los eventos adversos relacionados con las alteraciones de la NaS.

El resultado principal fue el cambio en la $\mathrm{NaS}$ (en $\mathrm{mmol} / \mathrm{L}$ ) entre el valor inicial y final producido por la infusión de las SM. La NaS se midió antes de la aleatorización y cuando los pacientes alcanzaron $>20 \%$ de la ingesta total de líquidos por vía oral o al cumplir 48 horas del inicio de la infusión de la SM, ya que la mayoría de los pacientes internados en la unidad alcanzan la alimentación enteral completa en las primeras 48 horas desde el ingreso o se les indica nutrición parenteral. La NaS y los niveles de glucosa se determinaron con un analizador de gases y electrolitos en sangre (Gem Premier 3000, Instrumentation Laboratory, Lexington, MA, EE.UU.). Se consideraron valores normales de NaS 135-145 mmol/L, hiponatremia <135 $\mathrm{mmol} / \mathrm{L}$, hiponatremia grave $<130 \mathrm{mmol} / \mathrm{L} \mathrm{e}$ hipernatremia $>145 \mathrm{mmol} / \mathrm{L}$.

Los resultados secundarios incluyeron la mortalidad a los 28 días después de la asignación 
aleatoria, el número de días sin ventilación mecánica a los 28 días desde el ingreso en la UCIP, la duración de la estadía en la UCIP medida en días, la presencia de hiponatremia e hipernatremia, y el número de pacientes que presentaron hiponatremia grave.

Dos observadores externos realizaron un análisis de los resultados cuando se alcanzó el reclutamiento de la mitad de la población, sin encontrar riesgos para completar el estudio.

Este estudio fue aprobado por la Junta de revisión institucional dependiente de Docencia e Investigación y el Comité de Bioética (NCT 01301274).

\section{Cálculo del tamaño muestral}

El cálculo del tamaño muestral se basó en el resultado principal del estudio. El descenso mínimo estimado de la $\mathrm{NaS}$ fue de $2 \mathrm{mmol} / \mathrm{L}$ en los pacientes con $\mathrm{SMH}$, con una desviación estándar de $2 \mathrm{mmol} / \mathrm{L}^{12}$ Teniendo en cuenta un error alfa de 0,05 y una potencia del $90 \%$ para detectar esta diferencia, el tamaño de la muestra se calculó en 30 pacientes por rama más las pérdidas por seguimiento.

\section{Aleatorización}

Los pacientes fueron asignados a recibir $\mathrm{SMH}$ o SMI a partir de una lista de aleatorización generada por computadora (Microsoft Excel 2007) en bloques de cuatro. El código de aleatorización fue mantenido por el farmacéutico investigador y ocultado a todo el personal encargado de la indicación y prescripción de las SM. Estas se prepararon y codificaron en la farmacia, y todos los participantes, enfermeras, miembros del personal médico y de investigación, los investigadores (excepto los farmacéuticos), y los miembros del comité de monitoreo de seguridad desconocían la concentración de sodio en las diferentes soluciones.

\section{Análisis estadístico}

Los datos continuos se expresaron como media \pm desviación estándar (DE) o mediana y rango intercuartil (IQ) y se compararon mediante la prueba de la $t$ de Student o la prueba de la $U$ de Mann-Whitney (datos no paramétricos). Los datos categóricos se presentaron en proporciones y se compararon mediante la prueba de la $\chi^{2} \mathrm{o}$ la prueba exacta de Fisher. Durante el estudio, se aceptó un límite significativo $<0,05$. Los resultados se analizaron con el programa STATA 9.0 para Windows (Copyright 1984-2003 Stata Corporation 4905 Lakeway Drive College Station, TX, EE.UU.).

\section{RESULTADOS}

Entre el 1 de febrero y el 31 de octubre de 2011, 251 pacientes fueron ingresados en la UCIP del HGNPE, 94 fueron elegibles para este estudio, y por último, 66 pacientes pediátricos fueron aleatorizados. En 3 pacientes la segunda medida de $\mathrm{NaS}$ no se pudo obtener y se los eliminó para el análisis final de datos. Quedaron incluidos en el estudio 63 pacientes (Figura 1). Treinta y dos pacientes fueron asignados en forma aleatorizada para recibir SMH y 31, para recibir SMI.

No hubo diferencias entre los grupos en cuanto a características basales: edad, sexo, peso, índice de mortalidad pediátrica 2 (PIM 2), condición (enfermedad médica o quirúrgica) y $\mathrm{NaS}$ (Tabla 1).

No hubo diferencias entre los grupos en la duración y la cantidad de SM administrada (Tabla 2). Los pacientes recibieron menos SM que la tasa de mantenimiento tradicional $(100 \mathrm{ml} / \mathrm{kg} /$ día hasta $10 \mathrm{~kg}$ de peso), 37\% menos en el SMH y $23 \%$ menos en los pacientes que recibieron SMI. ${ }^{1}$

Después de la infusión de SM, hubo una diferencia significativa en la NaS entre ambos grupos: SMI $140 \pm 4,1$ contra SMH 137,8 $2 \pm 4,3$ $\mathrm{mmol} / \mathrm{L}, p=0,04$. El cambio absoluto en el nivel de sodio sérico fue mayor en los pacientes que recibieron SMH $(-1,8 \mathrm{mmol} / \mathrm{L}[\mathrm{IQ}-0,1 ;-3,66])$ en comparación con los que recibieron SMI $(0,9$ $\mathrm{mmol} / \mathrm{L}$ [IQ -2,8; 1,1]); sin embargo, la diferencia no fue significativa.

No hubo diferencias entre los grupos en el número de días sin ventilación mecánica, el tiempo de internación en la UCIP y la mortalidad a los 28 días. Los tres pacientes que murieron y recibieron todos $\mathrm{SMH}$ mantuvieron una concentración de sodio por encima de $130 \mathrm{mmol} / \mathrm{L}$ durante el período de estudio y la causa de la muerte no estuvo aparentemente relacionada con la infusión de SM (Tabla 3).

Ambas SM no aumentaron el riesgo de hiponatremia (SMH, RR 1,21; IC 95\% 0,36 a 4,12; SMI, RR 0,83; IC 95\% 0,24 a 2,81) o hipernatremia (SMH, RR 1,93; IC 95\% 0,19 a 19,69; SMI, RR 0,52; IC $95 \% 0,05$ a 5,24$)$.

Solo hubo un caso de hiponatremia grave (NaS $<130 \mathrm{mmol} / \mathrm{L}$ ) en un paciente que recibió $\mathrm{SMH}$. 


\section{DISCUSIÓN}

Los resultados de este ensayo clínico mostraron que la disminución del sodio en el suero fue mayor con el uso de SM hipotónicas y que el uso de SM isotónicas no produjo alteraciones en la $\mathrm{NaS}$ en pacientes pediátricos críticamente enfermos. Estos resultados fueron similares a los observados en un ensayo

Figura 1. Flujograma de los sujetos del estudio

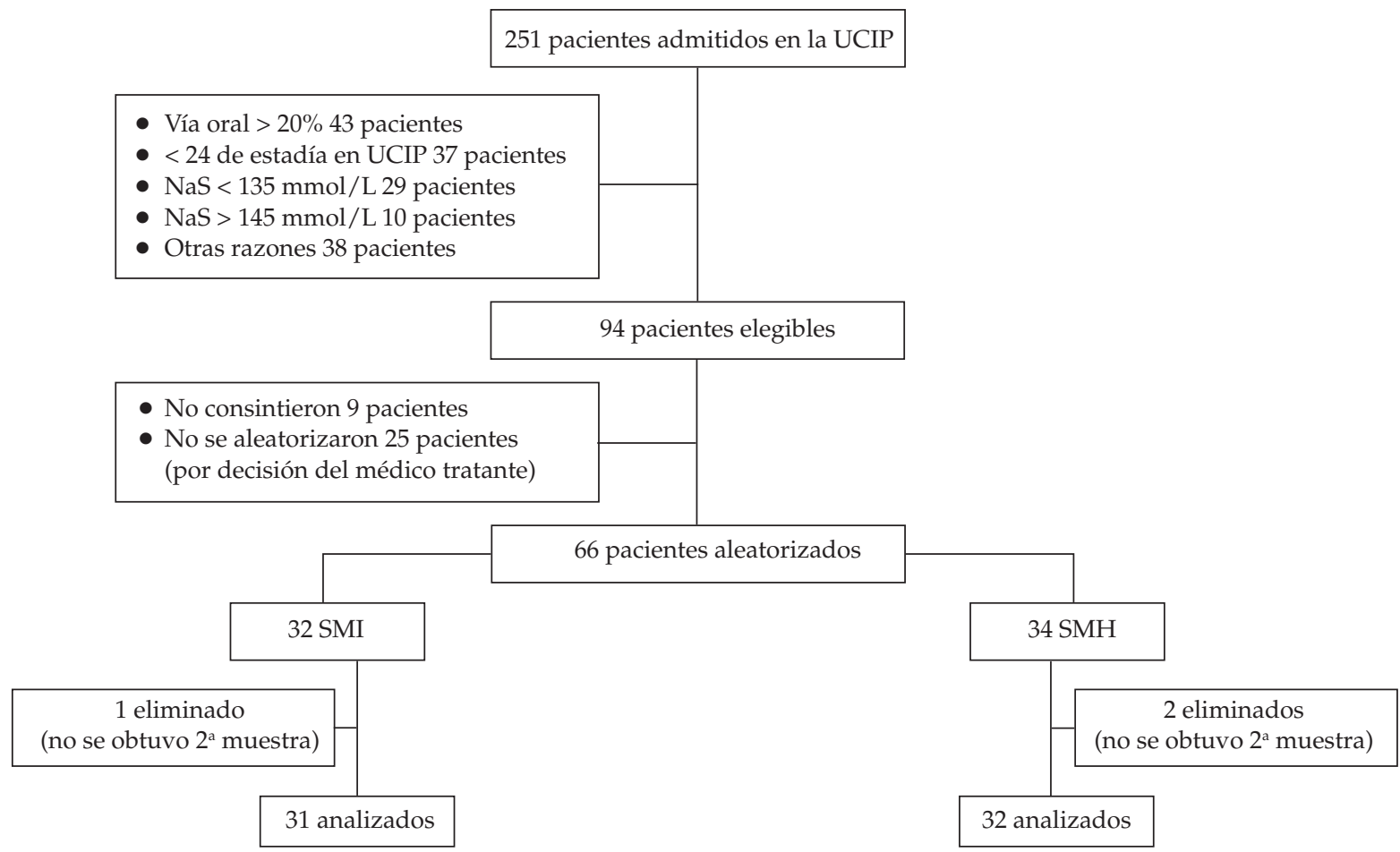

SMI: solución de mantenimiento isotónica; SMH: solución de mantenimiento hipotónica.

TABLA 1. Características de los pacientes al comienzo del estudio

\begin{tabular}{|c|c|c|c|c|}
\hline & Total & SMH & SMI & $p$ \\
\hline Pacientes aleatorizados $(n)$ & 63 & 32 & 31 & \\
\hline Varones $(n)(\%)$ & $37(58,7)$ & $18(56,2)$ & $19(61,3)$ & 0,80 \\
\hline Edad, mediana (IQ), (meses) & $5(3 ; 9)$ & $4,5(3 ; 9)$ & $5(3 ; 10)$ & 0,54 \\
\hline Peso, mediana (IQ), (kg) & $6(5 ; 11)$ & $6,1(5 ; 16)$ & $6(5 ; 10)$ & 0,35 \\
\hline PIM2, mediana (IQ) & $2,61(6 ; 7)$ & $4,4(1,4 ; 8,5)$ & $2,5(1,6 ; 7)$ & 0,73 \\
\hline \multicolumn{5}{|l|}{ Tipo de diagnóstico } \\
\hline Médico (total) (\%) & $59(89)$ & $29(85)$ & $30(93,5)$ & 0,71 \\
\hline Enfermedad respiratoria & $40(60)$ & $18(53)$ & $22(68)$ & 0,41 \\
\hline Enfermedad hematooncológica & $4(6)$ & $2(6)$ & $2(6,5)$ & 1 \\
\hline Enfermedad infecciosa & $9(14)$ & $5(15)$ & $4(12,5)$ & 0,73 \\
\hline Enfermedad neurológica & $6(9)$ & $4(11)$ & $2(6,5)$ & 0,42 \\
\hline Quirúrgico (total) (\%) & $7(11)$ & $5(15)$ & $2(6,5)$ & 0,42 \\
\hline $\mathrm{NaS}$ basal $($ media $\pm \mathrm{DE})(\mathrm{mmol} / \mathrm{L})$ & $139,4 \pm 3,4$ & $139,6 \pm 3,2$ & $139,2 \pm 3,5$ & 0,63 \\
\hline
\end{tabular}

PIM2: Pediatric Index of Mortality 2; NaS: concentración sérica de sodio; SMH: solución de mantenimiento hipotónica; SMI: solución de mantenimiento isotónica; IQ: intercuartil. 
realizado por Álvarez Montañana y cols. ${ }^{13} \mathrm{y}$ confirmados por un metanálisis ${ }^{11}$ que indica que la administración de SM hipotónica es un factor de riesgo importante para la producción de hiponatremia iatrogénica en pediatría. Un estudio reciente mostró una reducción de la hiponatremia en niños posoperados con el uso de SM isotónica, sin producción de hipernatremia iatrogénica. En este estudio, se observaron niveles elevados de la hormona antidiurética (ADH) en las primeras 24 horas después de estímulos no osmóticos para la secreción de $\mathrm{ADH} .{ }^{14}$

La disminución en la NaS puede deberse a un déficit de sodio o a un balance positivo de agua libre de electrolitos (ALE). Los niveles elevados de ADH afectan la excreción de ALE, y se han demostrado diferentes estímulos no osmóticos que aumentan la secreción de ADH. 5, , 14,15 Ambas SM indicadas a los pacientes en nuestro estudio contenían sodio suficiente para satisfacer las exigencias diarias ${ }^{1} \mathrm{y}$, por esa razón, la disminución de sodio sérico sería atribuible a la ingesta de ALE en exceso contenida en la SMH. La cantidad absoluta de la disminución de la $\mathrm{NaS}$ no fue clínicamente relevante en nuestro estudio, ya que el nivel de la natremia cayó por debajo de $130 \mathrm{mmol} / \mathrm{L}$ en un solo paciente, que no presentó manifestaciones clínicas.

Sin embargo, teniendo en cuenta que los pacientes de nuestro estudio recibieron menos SM que las cantidades originalmente descritas por Holliday y Segar en 1959, un 37\% menos en el SMH y $23 \%$ menos en el SMI, el descenso en la NaS pudo ser más importante con el $100 \%$ de la cantidad propuesta de SM por el enfoque tradicional. ${ }^{1}$ Álvarez Montañana y cols. sugirieron que la posibilidad de generar hiponatremia con la infusión de SM hipotónicas era acumulativa en función de la duración de la infusión. ${ }^{13}$

Kannan y cols. observaron una menor incidencia de hiponatremia cuando los pacientes recibieron dos tercios de las tasas de

TABLA 2. Cantidad de soluciones administradas, cambios en la concentración sérica de sodio y características de los pacientes al final del estudio

\begin{tabular}{lccc}
\hline & SMH $(n=32)$ & SMI $(n=31)$ & $p$ \\
\hline Soluciones administradas (mL) mediana (IQ) & $577(332 ; 987)$ & $594(336 ; 900)$ & 0,92 \\
Horas de administración de SM (media \pm DE) & $24 \pm 10,8$ & $27,6 \pm 12,8$ & 0,23 \\
Tasa (expresada como \% de la tasa tradicional de & & & \\
administración de soluciones de mantenimiento) & 63 & 77 & 0,04 \\
NaS final (mmol/L) (media \pm DE) & $137,8 \pm 4,3$ & $140 \pm 4,1$ & 0,40 \\
Días sin ventilación mecánica (media \pm DE) & $19,9 \pm 7,9$ & $21,5 \pm 6,6$ & 0,13 \\
Estadía en UCIP (días) (media \pm DE) & $8,5 \pm 5,5$ & $12 \pm 11,5$ & 0,08 \\
Mortalidad $(n)(\%)$ & $3(9,4)$ & $0(0)$ & 1 \\
Pacientes con hipernatremia $(n)(\%)$ & $2(6,7)$ & $1(3,3)$ & 1 \\
Pacientes con hiponatremia $(n)(\%)$ & $5(15,5)$ & $4(12,9)$ & \\
\hline
\end{tabular}

NaS: concentración sérica de sodio; SMH: solución de mantenimiento hipotónica; SMI: solución de mantenimiento isotónica; IQ: intercuartil; UCIP: unidad de cuidados intensivos pediátricos; SM: soluciones de mantenimiento.

TABLA 3. Características de los pacientes fallecidos

\begin{tabular}{|c|c|c|c|c|c|}
\hline Edad (meses) & Diagnóstico & $\mathrm{NaS}$ basal (mmol/L) & $\mathrm{NaS}$ final $(\mathrm{mmol} / \mathrm{L})$ & Día de la muerte & Causa de la muerte \\
\hline 46 & $\begin{array}{c}\text { Leucemia } \\
\text { linfoblástica aguda }\end{array}$ & 135 & 130 & 24 & Shock séptico \\
\hline 1 & Meningitis & 144 & 140 & 4 & Muerte cerebral \\
\hline 2 & $\begin{array}{c}\text { Infección por } \\
\text { Bordetella pertussis }\end{array}$ & 136 & 142 & 2 & Shock cardiogénico \\
\hline
\end{tabular}

Todos los pacientes recibieron soluciones de mantenimiento hipotónicas.

NaS: concentración sérica de sodio. 
mantenimiento estándar en comparación con las tasas estándares. ${ }^{16}$ Las SM hipotónicas dadas a tasas elevadas han sido relacionadas con la morbimortalidad en los pacientes pediátricos debido a la producción de hiponatremia iatrogénica. ${ }^{4,17-20}$ Algunos autores sugieren que el uso de menos volumen de SM sería igual o más eficaz en la prevención de la hiponatremia adquirida en el hospital que la administración de SM isotónicas. ${ }^{12,13,21}$ Neville y cols. hallaron que el riesgo de hiponatremia se redujo con el uso de SM isotónicas, pero no cuando se restringió la cantidad de SM administrada. ${ }^{22} \mathrm{El}$ presente estudio no fue formulado para comparar las variaciones en la $\mathrm{NaS}$ producidas por la administración de diferentes cantidades de SM.

La principal limitación de este estudio es la incapacidad para medir los niveles de ADH. Sin embargo, el aumento en los niveles de ADH en pacientes pediátricos hospitalizados se describió en diferentes estudios. ${ }^{5,8,14,15}$ En nuestro caso, se eliminaron pocos pacientes y todos tenían una medición de $\mathrm{NaS}$ al inicio del estudio. En muchos de los ensayos clínicos citados no se midió la NaS antes de la administración de SM y esto podría haber sobreestimado la presencia de hiponatremia causada por SM al final de la investigación. En nuestro estudio no se incluyeron pacientes con niveles séricos de sodio inferiores a $135 \mathrm{mmol} / \mathrm{L}$, y tal vez por esa razón no hemos visto manifestaciones clínicas o más pacientes con hiponatremia al terminar el estudio.

Solo en 3 pacientes se registró una natremia superior a $145 \mathrm{mmol} / \mathrm{L}$ y no fue de riesgo $(<150 \mathrm{mmol} / \mathrm{L})$. Debido a la criticidad de los pacientes fue difícil estimar los signos relacionados con la sobrecarga de sodio. Recientemente, la Agencia Nacional para la seguridad de los pacientes de Gran Bretaña emitió recomendaciones con respecto al uso de $\mathrm{SM}$, considerando de riesgo para el paciente cualquier concentración de sodio contenido en las SM menor de $0,45 \% .{ }^{23}$ Las SM con $\mathrm{NaCl}$ de $0,45 \%$ a $0,9 \%$ mostraron ser seguras en los estudios recopilados en una reciente revisión sistemática. ${ }^{14}$

Diferentes autores recomiendan obtener una medición de electrolitos antes de indicar una SM, y realizar controles de peso, balance de líquidos y control de parámetros clínicos estrictos durante su infusión. ${ }^{9,12,14}$

El período de intervención y la cantidad de SM administrada fueron pragmáticos, lo que permitió a los médicos ajustar los volúmenes infundidos y el momento de cambiar a la vía oral. Este tipo de intervención es muy similar a la atención habitual. Los resultados de los estudios específicos fueron enmascarados completamente para evitar el efecto Hawthorne.

\section{CONCLUSIONES}

Este estudio mostró que ninguna de las SM estudiadas produjo hiponatremia iatrogénica, a este ritmo de infusión, en 24 horas. La SM isotónica no disminuyó la NaS. Tampoco se observaron diferencias entre ambas SM en la producción de hipernatremia, número de días sin ventilación mecánica, tiempo de internación en la UCIP y mortalidad a los 28 días.

\section{Agradecimientos}

A los profesionales de la UCIP del Hospital "Pedro de Elizalde", a la Jefa del Sector Farmacia del HGNPE Susana Bengoa y al Dr. Fernando Ferrero por su apoyo durante el estudio y la revisión del manuscrito.

\section{BIBLIOGRAFÍA}

1. Holliday MA, Segar ME. The maintenance need for water in parenteral fluid therapy. Pediatrics 1957;19:823-32.

2. Carpenter J, Weinstein S, Myseros J, Vezina G, et al. Inadvertent hyponatremia leading to acute cerebral edema and early evidence of herniation. Neurocrit Care 2007;6(3):195-9.

3. Moritz ML, Ayus JC. Prevention of hospital-acquired hyponatremia: a case for using isotonic saline. Pediatrics 2003;111:227-30.

4. Arieff A, Ayus J, Fraser C. Hyponatremia and death or permanent brain damage in healthy children. BMJ 1992;304:1218-22.

5. Powell KR, Sugarmann LI, Eskanazi AE, Woodin KA, et al. Normalization of plasma arginine vasopressin concentrations when children with meningitis are given maintenance plus replacement fluid therapy. J Pediatr 1990;117(4):515-22.

6. Burrows F, Shutack JG, Crone R. Inappropriate secretion of antidiuretichormone in a postsurgical pediatric population. Intensive Care Med 1983;11:527-31.

7. Thomas TH, Morgan DB. Post-surgical hyponatremia: the role of intravenous fluids and arginine vasopressin. $\mathrm{Br} J$ Surg 1979;66:540-2.

8. Neville K, Verge C, Rosenberg A, O'Meara MW, et al. Isotonic is better than hypotonic saline intravenous rehydration of children with gastroenteritis: a prospective randomized study. Arch Dis Child 2006;91:226-32.

9. Hoorn EJ, Geary D, Robb M, Halperin ML, et al. Acute hyponatremia is related to intravenous fluid administration in hospitalized children: an observational study. Pediatrics 2004;113:1279-84.

10. Eulmesekian PG, Pérez A, Minces P, Bohn D. Hospitalacquired hyponatremia in postoperative pediatrics patients: Prospective observational study. Pediatr Crit Care Med 2010;4(11):479-83.

11. Choong K, Kho ME, Menon K, Bohn D. Hypotonic versus isotonic saline hospitalized children: a systematic review. Arch Dis Child 2006;91:828-35. 
12. Jorro Barón F, Balladores C, Carretero P, Lombardo L, et al. Efectos sobre la natremia por la administración de soluciones endovenosashipotónicasenniñoshospitalizados con infección respiratoria aguda baja. Arch Argent Pediatr 2009;107(4):335-9.

13. Álvarez Montañana P, Alapont M, Pérez Ocón A, López $\mathrm{PO}$, et al. The use of isotonic fluid as maintenance therapy prevents iatrogenic hyponatremia in pediatrics: A randomized, controlled open study. Pediatr Crit Care Med 2008;9(6):589-97.

14. Choong K, Arora S, Cheng J, Farrokhyar F, et al. Hypotonic versus isotonic maintenance fluids after surgery for children: A randomized controlled trial. Pediatrics 2011;128(5):857-66.

15. Brazel P, Mc Phee I. Inapropiate secretion of antidiuretic hormone in postoperative scoliosis patients: the role of fluid management. Spine (Phila Pa 1976) 1996;21(6):724-7.

16. Kannan L, Lodha R, Vivekanandhan S, Bagga A, et al. Intravenous fluid regimen and hyponatremia among children: a randomized controlled trial. Pediatr Nephrol 2010;25(11):2303-9.
17. Moritz M, Ayus J. Preventing neurological complications from dysnatremias in children. Pediatr Nephrol 2005;20(12):1687-700.

18. Halberthal M, Halperin M, Bohn D. Lesson of the week: Acute hyponatremia in children admitted to hospital. BMJ 2001;322:780-2.

19. Hanna S, Tibby S, Durward A, Murdoch IA. Incidence of hyponatremia and hyponatremic seizures in severe respiratory syncytial virus bronchiolitis. Acta Paediatr 2003;92:430-4.

20. Playfor S. Fatal iatrogenic hyponatremia. Arch Dis Child 2003;88:646-7.

21. Yung M,KeelyS. Randomized controlled trial of intravenous maintenance fluids. J Pediatr Child Health 2009;45(1-2):9-14.

22. Neville K, Sandeman D, Rubinstein A, Henry GM, et al. Prevention of hyponatremia during maintenanceintravenous fluid administration: a prospective randomized study of fluid type versus fluid rate. J Pediatr 2010;156(2):313-9.

23. Suggested template for local development of intravenous fluid guidelines. [Consulta: 11/07/2011]. Disponible en: www.npsa.nhs.uk/health/alerts. 